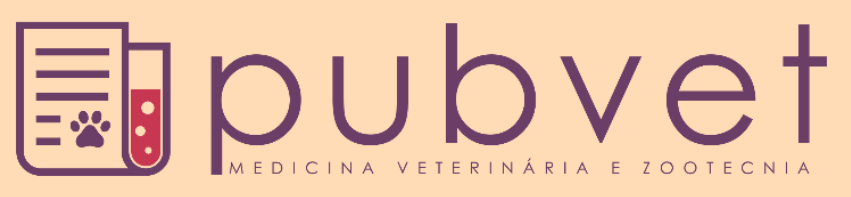

ISSN 1982-1263

https://doi.org/10.31533/pubvet.v13n2a266.1-7

\title{
Ciatostomíneos: uma revisão sobre a biologia, importância clínica e controle
}

\author{
Natália Soares Martins ${ }^{1^{*}}$, Diego Moscarelli Pinto $^{2} \bullet$, Laís Leal da Cunha ${ }^{\circ} \bullet$, Julia \\ Somavilla Lignon ${ }^{\circ}$, Tainá Ança Evaristo Mendes $\operatorname{Cardoso}^{4}{ }^{\circ}$, Alice Mueller $^{4} \odot$, Felipe \\ Geraldo Pappen ${ }^{2}{ }^{\circ}$, Leandro Quintana Nizoli² ${ }^{\circ}$ \\ ${ }^{I}$ Médica veterinária, doutoranda em Parasitologia da Universidade Federal de Pelotas, Departamento de Microbiologia e \\ Parasitologia. Pelotas - RS Brasil. \\ ${ }^{2}$ Professor da Universidade Federal de Pelotas, Departamento de Veterinária Preventiva. Pelotas - RS Brasil. \\ ${ }^{3}$ Médica veterinária, Pelotas - RS Brasil. \\ ${ }^{4}$ Acadêmica da Faculdade de Veterinária da Universidade Federal de Pelotas. Pelotas - RS Brasil. \\ *Autor para correspondência, E-mail: nataliasmartins@outlook.com
}

\begin{abstract}
Resumo. Os membros da subfamília Cyathostominae são conhecidos como ciatostomíneos ou "pequenos estrôngilos". Estes compõem um grupo de 50 espécies que podem parasitar equídeos, e são os parasitos mais prevalentes e resistentes aos anti-helmínticos em equinos jovens e adultos. Os ciatostomíneos comprometem o peristaltismo e a conversão alimentar, formando nódulos na parede do trato gastrintestinal a cada mudança de estádio larval. Além disso, podem ocasionar a Ciatostominose larval, uma síndrome clínica associada a emergência maciça de larvas encistadas. O controle destes parasitos é feito por meio da administração de anti-helmínticos, porém a ocorrência de resistência dos ciatostomíneos frente aos antiparasitários é uma realidade. Para a obtenção de um controle anti-helmíntico eficiente é fundamental o acompanhamento coproparasitológico e médico veterinário, com a realização de testes de eficácia periódicos, para que se possam estabelecer estratégias de controle adequadas para cada propriedade.
\end{abstract}

Palavras chave: pequenos estrôngilos, equinos, Ciatostominose, controle integrado

\section{Cyathostomins: a review of biology, clinical significance and control}

\begin{abstract}
Members of the subfamily Cyathostominae, also known as cyathostomins or "small strongyles". These are composed of a group of 50 species that can parasite horses, and are considered the most prevalent and resistant to anthelmintics parasites in young and adult horses. Cyathostomins may be compromised to produce a diet, forming nodules in experimental gastroenteritis. In addition, could cause larval cyathostominosis, a clinical syndrome associated with a massive emergency of encysted larvae. The control of these parasites is done through the administration of anthelmintics, but is frequent the occurrence of resistance. In order to obtain an effective anthelmintic control, it is essential the coproparasitological and veterinary monitoring, with periodic effectiveness tests, so that adequate control strategies can be established for each property.
\end{abstract}

Keywords: small strongyles, horses, cyathostominosis, strategic control

\section{Ciatostomas: una revisión de la biología, importancia clínica y control}

Resumen. Los miembros de la subfamilia Cyathostominae se conocen como ciatostomas o "pequeños estróngilos". Estos componen un grupo de 50 especies que pueden parasitar équidos, y son los parásitos más prevalentes y resistentes a los antiparasitarios en equinos 
jóvenes y adultos. Los ciatostomas comprometen el peristaltismo y la conversión alimenticia, formando nódulos en la pared del tracto gastrointestinal a cada cambio de estadio larval. Además, pueden ocasionar un síndrome clínico asociado a la salida masiva de larvas enquistadas. El control de estos parásitos se realiza por medio de la administración de antiparasitarios, pero la ocurrencia de resistencia a esos es una realidad. Para la obtención de un control antiparasitario eficiente es fundamental el acompañamiento coprológico, parasitológico y médico veterinario, con la realización de pruebas de susceptibilidad antiparasitaria frecuentes, para que se puedan establecer estrategias de control adecuadas para cada propiedad.

Palabras clave: pequeños estróngilos, equinos, control integrado

\section{Introdução}

Os estrongilídeos (ciatostomíneos e grandes estrôngilos) são parasitos comumente encontrados no intestino grosso de equinos e podem causar diversos impactos à saúde e bem-estar de seus hospedeiros. Estes parasitos são a principal razão pela qual os equinos de campo devem receber tratamento antihelmíntico regularmente. Durante décadas, os grandes estrôngilos, em especial Strongylus vulgaris, foram considerados como uma grande ameaça a vida dos equinos, sendo alvo dos programas de controle parasitário. Estes métodos conseguiram reduzir satisfatoriamente a população de $S$. vulgaris (Herd, 1990). Entretanto, possibilitaram o aumento da prevalência dos ciatostomíneos, especialmente devido à capacidade de adaptação destes às novas moléculas e tipos de tratamentos (Kaplan \& Nielsen, 2010). Atualmente é consenso geral que os ciatostomíneos são as principais espécies de parasitos dos equinos (Nielsen, 2012).

Os ciatostomíneos (Nematoda: Cyathostominae) são conhecidos popularmente como pequenos estrôngilos, compondo um grupo de 51 espécies que podem parasitar equídeos (Lichtenfels et al., 2008). Os equinos se infectam ao ingerir as formas infectantes presentes no ambiente, este processo se inicia logo após o nascimento por meio do contato direto ou indireto dos potros com outros animais jovens e/ou com suas mães. Os ciatostomíneos podem causar redução de crescimento, perda de peso, debilidade, diarreia e cólica. Destaca-se a ocorrência de enterite granulomatosa e a ciatostominose larval, doenças potencialmente fatais, associadas ao desenvolvimento e/ou emergência de grande número de estádios imaturos na parede do intestino grosso (Peregrine et al., 2006; Pierezan et al., 2009).

Embora diversos trabalhos tenham descrito a prevalência e os impactos dos ciatostomíneos na saúde equina, uma breve revisão de sua biologia e importância clínica pode ser de grande valia para entender a necessidade de desenvolver estratégias de controle parasitário apropriadas e eficazes, dessa forma contribuindo para melhor proteger os equinos destes parasitos.

\section{Biologia e epidemiologia}

Os ciatostomíneos são nematódeos pequenos (5 a $12 \mathrm{~mm}$ de comprimento) e com coloração avermelhada; sendo visíveis na inspeção da mucosa do intestino grosso ou até mesmo, em infecções severas, no bolo fecal. A cápsula bucal é pequena, cilíndrica e sem presença de dentes; justamente pelas características desta estrutura que a diferenciação das espécies é realizada (Lichtenfels et al., 2008).

\section{Ciclo biológico}

O ciclo de vida dos ciatostomíneos é direto e sem migrações complexas. As fêmeas adultas de ciatostomíneos realizam postura de ovos que são eliminados juntamente com as fezes dos equinos parasitados. Os ovos desenvolvem-se no ambiente, passando por dois estádios larvais intermediários até atingir a fase infectante. Os equinos se infectam ao ingerir, juntamente com a pastagem, larvas de terceiro estádio $\left(\mathrm{L}_{3}\right)$. Após a deglutição, as $\mathrm{L}_{3}$ penetram nas criptas de Lieberkuhn do ceco e cólon, atravessando as células da porção distal das criptas, localizando-se na mucosa ou submucosa. Ocorre a mudança para larvas de quarto estádio $\left(\mathrm{L}_{4}\right)$, durante o seu desenvolvimento são envolvidas por fibroblastos, os quais formam na submucosa, cistos fibrosos. Posteriormente, as $\mathrm{L}_{4}$ migram para o lúmen do intestino grosso e mudam para larvas de quinto estádio $\left(\mathrm{L}_{5}\right)$, estas após completarem seu desenvolvimento dão origem aos adultos (Reinemeyer \& Nielsen, 2013). 
O período pré-patente possui entre seis a 18 semanas, entretanto pode ser prolongado devido ao fenômeno da hipobiose. Esta é uma fase de inibição temporária do desenvolvimento larvar e tem importante papel na patogenia da parasitose (Lyons et al., 2000). As larvas podem permanecer em hipobiose por até dois anos, retomando o seu desenvolvimento quando as condições externas tornam-se favoráveis (Corning, 2009).

\section{Epidemiologia}

Os ciatostomíneos são os parasitos mais prevalentes e com maior intensidade parasitária em equinos no Brasil, representando de 80-100\% da carga parasitária total (Barbosa et al., 2001; Pereira \& Vianna, 2006). São encontrados mais comumente em animais criados a campo, embora equinos estabulados também possam ser parasitados. Este fato pode ser justificado, pois no interior dos estábulos as fezes secam mais rápido e mesmo quando há umidade adequada, o alto teor de amônia, proveniente da urina, é tóxico para o desenvolvimento larvar, não proporcionando um microclima adequado para o desenvolvimento até a forma infectante (Reinemeyer \& Nielsen, 2013).

Nos meses mais quentes do ano observa-se maior número de larvas infectantes no pasto devido as condições ambientais favoráveis para o desenvolvimento do ovo até $\mathrm{L}_{3}$, este fato pode intensificar as infecções no outono (Taylor et al., 2017). Além disso, existem evidências de que, em locais com clima subtropical, muitas $\mathrm{L}_{3}$ de ciatostomíneos ingeridas durante o outono entram em hipobiose e permanecem na mucosa do intestino grosso até a primavera seguinte; a emergência em massa destas larvas pode acarretar em diarreia grave (Love et al., 1999; Lyons et al., 2000; Peregrine et al., 2006).

\section{Importância clínica}

As infecções por ciatostomíneos normalmente são bem toleradas pelo hospedeiro; porém estes parasitos podem causar enteropatia inflamatória que afeta o ceco e o cólon (Love et al., 1999). A penetração das $\mathrm{L}_{3}$ na mucosa intestinal e a grande quantidade de larvas encistadas na parede do intestino grosso podem acarretar em enterite granulomatosa, cursando com diarreia, pirexia, taquicardia, taquipneia e emagrecimento progressivo dos animais (Pierezan et al., 2009).

O fenômeno da hipobiose também tem importância na patogenicidade dos ciatostomíneos, já que as larvas encistadas ficam protegidas do sistema imunológico do hospedeiro e da ação da maioria dos antihelmínticos utilizados. A maturação e emergência em massa das larvas encistadas (com consequente destruição da mucosa e inflamação severa local) acarreta em fraqueza, diarreia grave, desidratação, perda de peso acentuada, edema subcutâneo e cólica, podendo por vezes ser fatal (Peregrine et al., 2006; Pierezan et al., 2009). Este fenômeno é chamado de Ciatostominose tipo II ou Ciatostominose larval (Love et al., 1999; Lyons et al., 2000) e pode gerar algumas anormalidades nas análises clínicas, tais como: hipoalbuminemia, hipoproteinemia, microcitose e elevação dos níveis de fibrinogênio (Peregrine et al., 2006). A Ciatostominose larval é normalmente diagnosticada em equinos de um a três anos de idade no final do inverno ou início da primavera, entretanto a suscetibilidade aos ciatostomíneos perdura por toda a vida dos hospedeiros, dessa maneira a doença clínica pode ocorrer em equinos de qualquer idade e em qualquer estação do ano, podendo ocasionar o óbito em até 50\% dos casos (Love et al., 1999). A inespecificidade dos sinais clínicos pode dificultar o diagnóstico; porém a história clínica do paciente e a exclusão de outras possíveis causas podem auxiliar na obtenção do diagnóstico presuntivo. Em alguns casos, grande quantidade de $\mathrm{L}_{4}$ e/ou ciatostomíneos adultos podem ser encontradas nas fezes, dando suporte a uma suspeita de ciatostominose larval (Peregrine et al., 2006).

\section{Controle}

Sem dúvida, o controle da verminose equina resulta em melhor desempenho dos animais. Na maioria dos planteis utilizam-se intensamente os compostos anti-helmínticos por sua praticidade, eficiência e segurança (Duarte et al., 2008). O controle tradicional baseia-se principalmente na aplicação de antihelmínticos de três principais classes de medicamentos: os benzimidazóis (fembendazol e oxibendazol), a tetrahidropirimidina (pirantel) e as lactonas macrocíclicas (ivermectina e moxidectina) (von SamsonHimmelstjerna, 2012). Sendo que no mercado global equino as lactonas macrocíclicas são as mais amplamente utilizadas (Allison et al., 2011; Nielsen et al., 2006). 
Uma vez que equinos de qualquer idade podem se tornar infectados e excretar ovos, todos os animais a pasto com mais de dois meses de idade podem ser tratados, preferencialmente de acordo com o resultado do exame coprológico. Os benzimidazóis, o pirantel e as lactonas macrocíclicas são efetivos na remoção dos ciatostomíneos que vivem no lúmen. Todavia, nenhum composto antiparasitário é eficaz contra todos os estádios de desenvolvimento dos parasitos de equinos (Molento, 2005), sendo que somente a moxidectina tem efeito moderado contra larvas encistadas de terceiro e quarto estádio e nenhum efeito contra larvas em hipobiose (Monahan et al., 1996). Historicamente, o controle químico dos parasitos gastrintestinais era realizado de forma supressiva ou curativa. $\mathrm{O}$ tratamento curativo visa tratar os equinos que apresentam sinais clínicos relacionados a infecções por endoparasitos (Sangster, 2003), este método pode não impedir os efeitos deletérios dos parasitos gastrintestinais frente aos hospedeiros, aumentando o risco de óbito de animais. Já o tratamento supressivo consiste na aplicação do medicamento a cada quatro a oito semanas visando interromper o ciclo de vida dos parasitos (Molento, 2005), método amplamente utilizado desde a década de 1960 nos EUA (Kaplan \& Nielsen, 2010). Como consequência do tratamento supressivo, especialmente das administrações frequentes de antiparasitários, houve a promoção da seleção de organismos resistentes (Molento, 2005), problema que se encontra disseminado nos planteis de equinos (Kaplan et al., 2004; Molento et al., 2008; Romero et al., 2014; Traversa et al., 2009). Atualmente, ciatostomíneos resistentes a anti-helmínticos são altamente prevalentes e mesmo quando as drogas ainda são efetivas, o período de reaparecimento de ovos após o tratamento tem sido drasticamente reduzido (Lyons et al., 2008; von Samson-Himmelstjerna, 2012).

\section{Resistência anti-helmíntica}

A redução da eficácia dos antiparasitários tornou-se uma séria ameaça à saúde e à produção animal em diversas localidades. A taxa de desenvolvimento da resistência aos anti-helmínticos é determinada pela pressão de seleção e consequente passagem de genes dos organismos sobreviventes ao tratamento para a próxima geração. Muitos nematódeos de importância veterinária têm características genéticas que favorecem o desenvolvimento deste processo (Hodgkinson et al., 2008), e dentre os que parasitam os equídeos, os ciatostomíneos são os que apresentam maior capacidade de adaptação às novas moléculas e tipos de tratamentos anti-helmínticos (Love \& Duncan, 1991).

O primeiro relato da ocorrência de resistência de ciatostomíneos frente ao benzimidazol foi realizado por Slocombe \& Cote (1977), e desde então a redução da eficácia a este princípio ativo já foi registrada em mais de 14 países, sendo considerado o tipo mais comum e difundido de resistência (Peregrine et al., 2006). Nas últimas décadas diversos estudos demonstraram a redução da eficácia dos benzimidazóis, do pamoato de pirantel e até mesmo das lactonas macrocíclicas (Canever et al., 2013; Kaplan et al., 2004; Molento et al., 2008; Traversa et al., 2009).

\section{Avaliação da eficácia dos fármacos anti-helmínticos}

O monitoramento da eficácia das bases químicas é imprescindível para um programa de controle parasitário eficaz na propriedade, visando detectar a reduzida eficácia das drogas em populações de nematódeos. Para esse fim, diversos métodos e abordagens diferentes podem ser aplicados. O ensaio padrão é o Teste de Redução de Contagem de Ovos Fecais (TRCOF), que se baseia na quantificação de ovos por gramas de fezes (OPG) no pré e pós-tratamento dos equinos (Coles et al., 1992). Este método é o mais adequado para avaliar a eficácia de todas as classes de medicamentos disponíveis no mercado (Kaplan \& Nielsen, 2010; von Samson-Himmelstjerna, 2012). De modo geral, consideram-se como critérios para a suspeita de resistência, resultados do TRCOF inferiores a 95\% e com limite inferior do intervalo de confiança abaixo de 90\% (Coles et al., 1992). Kaplan \& Nielsen (2010) sugeriram o estabelecimento de valores mínimos específicos para cada classe de medicamentos, sendo: $90 \%$ para benzimidazóis, $90 \%$ para pirantel e $95 \%$ para as lactonas macrocíclicas. De qualquer maneira, a eficácia dos anti-helmínticos deve ser monitorada, no mínimo anualmente, em todos equinos da propriedade, ou numa amostra representativa no caso de grandes populações (Reinemeyer \& Nielsen, 2013)

\section{Controle integrado dos ciatostomíneos}

Nos últimos anos, tornou-se clara a necessidade de uma nova abordagem integrada no controle dos parasitos, como por exemplo, o método de tratamento seletivo, além de outras medidas de manejo que 
reduzam a infecção dos hospedeiros, bem como a pressão de seleção aos parasitos (Nielsen, 2012; Nielsen, 2015). Independentemente do método empregado, o monitoramento da eficácia dos produtos utilizados é fundamental para o sucesso do programa antiparasitário na propriedade.

Tratamento seletivo: Nos últimos anos, vários autores têm defendido que a vermifugação dos animais deve ser realizada de maneira seletiva, visto que, o uso indiscriminado e/ou inadequado dos antihelmínticos, pode contribuir no desenvolvimento da resistência anti-helmíntica (Coles et al., 1992; Nielsen, 2015; Schneider et al., 2014). O tratamento seletivo baseia-se no fato de que em uma população de equinos, apenas uma pequena porção dos animais alberga a maior parte dos parasitos, sendo estes os maiores contaminadores do ambiente; o restante dos animais tem uma carga parasitária baixa que se mantem praticamente inalterada, mesmo quando os animais não sofrem tratamento anti-helmíntico (Kaplan \& Nielsen, 2010). Além disso, a eliminação de ovos de estrongilídeos nas fezes é individual e segue um padrão consistente, o que significa que um equino que elimina muitos ovos num determinado momento, permanecerá assim durante toda a sua vida, e vice-versa (Becher et al., 2010). Este fato decorre da diferente susceptibilidade individual dos equinos aos parasitos.

Diante do exposto, faz sentido tratar apenas os equinos com maior nível de infecção parasitária, ou seja, aqueles que eliminam maior quantidade de ovos pelas fezes. A partir do método de Gordon \& Whitlock (1939) modificado pode-se determinar o OPG (Ovos Por Grama de fezes), permitindo averiguar quais equinos estão parasitados e classificar o nível de infecção parasitária (Kuz'mina, 2012; Nielsen et al., 2010). Dessa maneira, o tratamento só é realizado a partir de um determinado valor de OPG, denominado "cut-off" ou ponto de corte (Kaplan \& Nielsen, 2010; Reinemeyer, 1986; Reinemeyer \& Nielsen, 2013; Schneider et al., 2014). Segundo Nielsen (2012), o valor do "cut-off" pode situar-se entre 0-500 OPG, sendo que os animais que apresentem um valor de OPG abaixo do determinado como "cut-off", não são vermifugados.

Manejo de pastagens:As formas de vida livre dos ciatostomíneos se encontram especialmente nas pastagens, constituindo risco de infecção aos hospedeiros. Dessa maneira, são necessárias medidas de manejo que permitam reduzir a contaminação ambiental e consequentemente à infecção parasitária (Nielsen, 2012). A aração e gradagem dos piquetes pode ser uma alternativa para reduzir a quantidade de larvas infectantes nas pastagens, especialmente na época mais quentes do ano para favorecer o dessecamento das L 3 (Reinemeyer, 1986; Reinemeyer \& Nielsen, 2013). Pode ser também efetuada a rotação das pastagens, com intervalo de vários meses, visando quebrar os ciclos de vida parasitários (Van Doorn et al., 2012). O pastoreio em conjunto com espécies de ruminantes, principalmente com bovinos, também é benéfico na redução da contaminação ambiental e da exposição dos cavalos aos parasitos (Matthews, 2014; Nielsen, 2012). Este método tem o intuito de eliminar as formas infectantes, fato que ocorre após a ingestão das mesmas por uma espécie diferente, já que os ciatostomíneos tem especificidade de hospedeiros, parasitando somente equídeos. Além disso, o excesso populacional dos piquetes aumenta a propensão para o parasitismo intestinal por estrongilídeos, especialmente nos equinos criados sob manejo extensivo (Reinemeyer, 1986; Reinemeyer \& Nielsen, 2013). Portanto, a densidade animal nos piquetes deve ser reduzida para evitar que os equinos pastoreiem em zonas contaminadas com fezes e, portanto, com parasitos (Van Doorn et al., 2012).

\section{Conclusão}

Nas últimas décadas diversos estudos possibilitaram um avanço no conhecimento de questões complexas da biologia, importância clínica e controle dos ciatostomíneos. Estes parasitos representam risco à saúde e ao bem-estar dos equinos e não devem ser negligenciados. Para a obtenção de um controle anti-helmíntico eficiente é fundamental o acompanhamento parasitológico e médico veterinário para que se possam estabelecer estratégias de controle adequadas para cada propriedade. Para tanto, sugerese que amostras de fezes devem ser examinadas a intervalos regulares para monitorar a eficácia dos fármacos. Além disso, métodos alternativos de controle devem estar associados ao o tratamento químico reduzindo o uso geral de anti-helmínticos, e consequentemente, a pressão de seleção para o desenvolvimento de parasitos resistentes.

\section{Referências bibliográficas}

Allison, K., Taylor, N. M., Wilsmore, A. J. \& Garforth, C. (2011). Equine anthelmintics: survey of the patterns of use, beliefs and attitudes among horse owners in the UK. Veterinary Record, 168(18):483-483. 
Barbosa, O. F., Rocha, U. F., Silva, G. S., Soares, V. E., Veronez, V. A., Oliveira, G. P., . . Costa, A. J. (2001). A survey on Cyathostominae nematodes (Strongylidea, Strongylidae) in pasture bred horses from São Paulo State, Brazil. Semina: Ciências Agrárias, 22(1):21-26.

Becher, A. M., Mahling, M., Nielsen, M. K. \& Pfister, K. (2010). Selective anthelmintic therapy of horses in the Federal states of Bavaria (Germany) and Salzburg (Austria): An investigation into strongyle egg shedding consistency. Veterinary Parasitology, 171(1-2):116-122.

Canever, R. J., Braga, P. R. C., Boeckh, A., Grycajuck, M., Bier, D. \& Molento, M. B. (2013). Lack of Cyathostomin sp. reduction after anthelmintic treatment in horses in Brazil. Veterinary Parasitology, 194(1):35-39.

Coles, G. C., Bauer, C., Borgsteede, F. H. M., Geerts, S., Klei, T. R., Taylor, M. A. \& Waller, P. J. (1992). World Association for the Advancement of Veterinary Parasitology (WAAVP) methods for the detection of anthelmintic resistance in nematodes of veterinary importance. Veterinary Parasitology, 44(1-2):35-44.

Corning, S. (2009). Equine cyathostomins: a review of biology, clinical significance and therapy. Parasites \& Vectors, 2(2):1-6.

Duarte, E. R., Oliveira, N. J. F., Silveira, J. T., Ribeiro, F. L. A. \& Souza, R. M. (2008). Controle de verminose em equinos no norte de Minas Gerais com associação de pamoato de pirantel e ivermectina. Revista Caatinga, 21(1):1-4.

Gordon, H. M. \& Whitlock, H. V. (1939). A new technique for counting nematode eggs in sheep faeces. Journal of the Council for Scientific and Industrial Research, 12(1):50-52.

Herd, R. P. (1990). The changing world of worms: The rise of the cyathostomes and the decline of Strongylus vulgaris. Compendium on Continuing Education for the Practicing Veterinarian, 12(5):732-736.

Hodgkinson, J. E., Clark, H. J., Kaplan, R. M., Lake, S. L. \& Matthews, J. B. (2008). The role of polymorphisms at $\beta$ tubulin isotype 1 codons 167 and 200 in benzimidazole resistance in cyathostomins. International Journal for Parasitology, 38(10):1149-1160.

Kaplan, R. M., Klei, T. R., Lyons, E. T., Lester, G., Courtney, C. H., French, D. D., . . Zhao, Y. (2004). Prevalence of anthelmintic resistant cyathostomes on horse farms. Journal of the American Veterinary Medical Association, 225(6):903-910.

Kaplan, R. M. \& Nielsen, M. K. (2010). An evidence-based approach to equine parasite control: It ain't the 60s anymore. Equine Veterinary Education, 22(6):306-316.

Kuz'mina, T. A. (2012). Strongylids (Nematoda: Strongylidae) of domestic horses in Ukraine: modern state of fauna and structure of the parasite community. Parazitologiia, 46(2):127-138.

Lichtenfels, J. R., Kharchenko, V. A. \& Dvojnos, G. M. (2008). Illustrated identification keys to strongylid parasites (Strongylidae: Nematoda) of horses, zebras and asses (Equidae). Veterinary Parasitology, 156(1-2):4-161.

Love, S. \& Duncan, J. L. (1991). Could the worms have turned? Equine Veterinary Journal, 23(3):152154.

Love, S., Murphy, D. \& Mellor, D. (1999). Pathogenicity of cyathostome infection. Veterinary Parasitology, 85(2-3):113-122.

Lyons, E. T., Drudge, J. H. \& Tolliver, S. C. (2000). Larval cyathostomiasis. Veterinary Clinics of North America: Equine Practice, 16(3):501-513.

Lyons, E. T., Tolliver, S. C., Ionita, M., Lewellen, A. \& Collins, S. S. (2008). Field studies indicating reduced activity of ivermectin on small strongyles in horses on a farm in Central Kentucky. Parasitology Research, 103(1):209-215.

Matthews, J. B. (2014). Anthelmintic resistance in equine nematodes. International Journal for Parasitology: Drugs and Drug Resistance, 4(3):310-315.

Molento, M. B. (2005). Resistência parasitária em helmintos de equídeos e propostas de manejo. Ciência Rural, 35(6):1469-1477.

Molento, M. B., Antunes, J., Bentes, R. N. \& Coles, G. C. (2008). Anthelmintic resistant nematodes in Brazilian horses. Veterinary Record, 162384-385. 
Monahan, C. M., Chapman, M. R., Taylor, H. W., French, D. D. \& Klei, T. R. (1996). Comparison of moxidectin oral gel and ivermectin oral paste against a spectrum of internal parasites of ponies with special attention to encysted cyathostome larvae. Veterinary Parasitology, 63(3-4):225-235.

Nielsen, M. K. (2012). Sustainable equine parasite control: perspectives and research needs. Veterinary Parasitology, 185(1):32-44.

Nielsen, M. K. (2015). Universal challenges for parasite control: a perspective from equine parasitology. Trends in Parasitology, 31(7):282-284.

Nielsen, M. K., Baptiste, K. E., Tolliver, S. C., Collins, S. S. \& Lyons, E. T. (2010). Analysis of multiyear studies in horses in Kentucky to ascertain whether counts of eggs and larvae per gram of feces are reliable indicators of numbers of strongyles and ascarids present. Veterinary Parasitology, 174(1-2):77-84.

Nielsen, M. K., Monrad, J. \& Olsen, S. N. (2006). Prescription-only anthelmintics-a questionnaire survey of strategies for surveillance and control of equine strongyles in Denmark. Veterinary Parasitology, 135(1):47-55.

Peregrine, A. S., McEwen, B., Bienzle, D., Koch, T. G. \& Weese, J. S. (2006). Larval cyathostominosis in horses in Ontario: an emerging disease? The Canadian Veterinary Journal, 47(1):80-82.

Pereira, J. R. \& Vianna, S. S. S. (2006). Gastrointestinal parasitic worms in equines in the Paraíba Valley, State of São Paulo, Brazil. Veterinary Parasitology, 140(3-4):289-295.

Pierezan, F., Rissi, D. R., Oliveira Filho, J. C., Lucena, R. B., Tochetto, C., Flores, M. M., . . Barros, C. S. L. (2009). Enterite granulomatosa associada a larvas de ciatostomíneos em eqüinos no Rio Grande do Sul. Pesquisa Veterinária Brasileira, 29(5):382-386.

Reinemeyer, C. R. (1986). Small strongyles. Recent advances. The Veterinary clinics of North America. Equine practice, 2(2):281-312.

Reinemeyer, C. R. \& Nielsen, M. K. (2013). Handbook of equine parasite control. Iowa, USA: WileyBlackwell.

Romero, J. S., Ponce, J. D. M., Llorens, Y. G. \& Suárez, J. C. M. (2014). Prevalencia de nematodos intestinales y eficacia de Labiomec ${ }^{\circledR}$ en caballos de Camagüey, Cuba. Revista de Salud Animal, 36(3):152-158.

Sangster, N. (2003). A practical approach to anthelmintic resistance. Equine Veterinary Journal, 35(3):218-219.

Schneider, S., Pfister, K., Becher, A. M. \& Scheuerle, M. C. (2014). Strongyle infections and parasitic control strategies in German horses-a risk assessment. BMC Veterinary Research, 10(262):1-9.

Slocombe, J. O. \& Cote, J. F. (1977). Small strongyles of horses with cross resistance to benzimidazole anthelmintics and susceptibility to unrelated compounds. The Canadian Veterinary Journal, 18(8):212-217.

Taylor, M. A., Coop, R. L. \& Wall, R. L. (2017). Parasitologia Veterinária. Rio de Janeiro, Brasil: Guanabara Koogan.

Traversa, D., von Samson-Himmelstjerna, G., Demeler, J., Milillo, P., Schürmann, S., Barnes, H., . . Beraldo, P. (2009). Anthelmintic resistance in cyathostomin populations from horse yards in Italy, United Kingdom and Germany. Paper presented at the Parasites \& Vectors.

Van Doorn, D. C. K., Eysker, M., Kooyman, F. N. J., Wagenaar, J. A. \& Ploeger, H. W. (2012). Searching for ivermectin resistance in Dutch horses. Veterinary Parasitology, 185(2-4):355-358.

von Samson-Himmelstjerna, G. (2012). Anthelmintic resistance in equine parasites-detection, potential clinical relevance and implications for control. Veterinary Parasitology, 185(1):2-8.

Recebido: 10 janeiro, 2019.

Aprovado: 1 fevereiro, 2019

Publicado: 15 fevereiro, 2019

Licenciamento: Este artigo é publicado na modalidade Acesso Aberto sob a licença Creative Commons Atribuição 4.0 (CCBY 4.0), a qual permite uso irrestrito, distribuição, reprodução em qualquer meio, desde que o autor e a fonte sejam devidamente creditados. 